\title{
Regime transitions of granular flow in a shear cell: A micromechanical study
}

\author{
X. Wang, ${ }^{1}$ H. P. Zhu, ${ }^{2}$ S. Luding, ${ }^{3}$ and A. B. $\mathrm{Yu}^{1, *}$ \\ ${ }^{1}$ Laboratory for Simulation and Modeling of Particulate Systems, School of Materials Science and Engineering, \\ University of New South Wales, Sydney, New South Wales 2052, Australia \\ ${ }^{2}$ School of Computing, Engineering and Mathematics, University of Western Sydney, Locked Bag 1797, Penrith, \\ New South Wales 2751, Australia \\ ${ }^{3}$ Multi Scale Mechanics, Faculty of Engineering Technology (CTW), MESA + , University of Twente, P.O. Box 217, \\ 7500 AE Enschede, Netherlands
}

(Received 30 December 2012; revised manuscript received 20 July 2013; published 10 September 2013)

\begin{abstract}
The regime transitions of granular flow in a model shear cell are investigated numerically with a stresscontrolled boundary condition. The correlations between the elastically and kinetically scaled stresses and the packing fraction are examined, and two packing fractions $(0.58$ and 0.50$)$ are identified for the quasistatic to intermediate and intermediate to inertial regime transitions. The profiles and structures of contact networks and force chains among particles in different flow regimes are investigated. It is shown that the connectivity (coordination number) among particles and the homogeneity in the shear flow increase as the system goes through the inertial, intermediate, and then quasistatic regimes, and there is only little variation in the internal structure after the system has entered the quasistatic regime. Short-range force chains start to appear in the inertial regime, which also depend on the magnitude of the shear rate. The percolation of system-spanning force chains through the whole system is a characteristic of the onset of the quasistatic regime, which happens at a packing fraction that is close to the glass transition, i.e., about random loose packing (0.58) but far below the isotropic quasistatic (athermal) jamming packing fraction of random close packing (0.64). The tails of the probability density distribution $P(f)$ of the scaled normal contact forces for the flows in different regimes are quantified by a stretched exponential $P(f)=\exp \left(-c f^{n}\right)$ with a remarkable finding that $n \sim 1.1$ may be a potential demarcation point separating the quasistatic regime and the inertial or intermediate regimes.
\end{abstract}

DOI: 10.1103/PhysRevE.88.032203

PACS number(s): 45.70.Mg, 47.57.Gc, 45.70.Vn

\section{INTRODUCTION}

Granular materials are widely encountered in nature and in industries. The transportation, storage, mixing, fluidization, and coating of solid powders are routine processes in industries, such as food, mining, chemical, and pharmaceutical. In these processes, particulate materials can display very complicated dynamic behavior due to both the complex interactions between constituent particles and their interactions with surrounding gas or liquid and walls [1]. Even though we consider the cases where the interstitial fluid plays an insignificant role and particle-particle interactions are predominant, a general theory of the dynamics of grain flows is still lacking [2]. Complexity in formulating such a theory arises partly from the fact that particle flows can fall into three distinct but interconnected regimes: quasistatic, intermediate, and inertial. In the past, much effort had been dedicated to studying the two ends of the spectrum. On one hand, slowly sheared particles are in close contact, and the resulting stresses are of quasistatic rate-independent Coulomb type. Continuum theory of elastoplasticity is one of the many possible examples that could be used to model the stresses in this regime $[3,4]$ but has serious shortcomings with respect to the smooth transition between (almost) elastic and perfectly plastic regimes [5,6]. On the other end, rapidly flowing assemblies of particles behave inertially, and constituent grains are likely to be in contact for a short time. The resemblance of particles in this highly

${ }^{*}$ Corresponding author: a.yu@unsw.edu.au agitated regime to gas molecules leads to the fact that the so-called kinetic theory is used to describe the particle flows in this regime [7-13]. Somewhere in-between the quasistatic and the inertial flow regimes, a transitional regime can be identified, which is also called the intermediate regime. The flow characteristics in this regime have been investigated in terms of dense kinetic theory [13]; their frictional-collisional [14-16] or elastic-inertial [17-19] nature is recognized as one of the most difficult problems in granular materials research [20,21]. Although various constitutive equations proposed in the literature (see, e.g., Ref. [6] and references therein) have achieved a certain extent of success in depicting some aspects of the corresponding regimes, they often become inapplicable when there is a flow regime change.

The understanding of regime transitions and a regime map for granular flows is essential in order to derive an accepted general constitutive relation, which is applicable to all flow regimes. There are a lot of studies concerning phase change in granular flows under two kinds of system-scale constraint: volume- or strain-controlled [17,22-25], stresscontrolled $[6,18,26]$ or mixed stress-strain-control modes, such as, e.g., triaxial tests [5]. The earliest paper devoted to granular flow mapping can be attributed to Babic et al. [22]. In their paper, they employed a discrete element method (DEM) to simulate a two-dimensional volume-controlled system of simple shear flow with inelastic monosized disks. They constructed a regime chart in the parametric space of the volume fraction-dimensionless shear rate and divided the space into three parts by considering the parameters of the coordination number $(\mathrm{CN})$ and collision frequency: quasistatic 
flow, rapid flow, and transitional flow. A subsequent and more comprehensive study in this field is due to Campbell [17], who investigated a three-dimensional (3D) volume-controlled system of simple shear flow with monosized, cohesionless, and frictional particles. He found four flow regimes: elastic quasistatic, elastic inertial, inertial collisional, and inertial noncollisional. Since different aspects of granular flow were examined in the determination of the flow regimes, the regime charts due to Babic et al. [22] and Campbell [17] reflected different fundamental physics. Campbell [18] attempted to link the two regime charts and found that the quasistatic regime in Ref. [22] did not correspond to any regime in Ref. [17] as the densities considered were totally different. Considering both volume-controlled and stress-controlled cases, Campbell [18] compared the results from both cases and drew the conclusion that there were significant differences between these two types of systems and that rheological properties obtained in the volume-controlled system might not be applied to stress-controlled systems or vice versa. However, this issue was closely examined and was placed in doubt later by Aarons and Sundaresan [23,26]. They investigated both volume-controlled and stress-controlled systems of simple shear flow with monosized, spherical, and cohesive particles and found that the rheological behavior of the particles was the same regardless of whether the system was stress controlled or volume controlled, and thus, the regime map was independent of the type of system constraint. In a subsequent paper by Campbell [27], the rheology of ellipsoidal particles under volume-controlled conditions was studied, but the difference between the two boundary conditions was not addressed.

Despite the useful conclusions drawn from these studies, there is still no consensus regarding how an entire flow regime map can be drawn and if a change in the system-scale constraint has an influence on the regime map. Of the two types of constraint, the stress-controlled condition is more relevant in reality as there is always free space in which granular flow can expand or compress in response to different stresses, and particles always have to support a certain amount of overburden. Exceptions are flows with a free surface [6] where the particles experience no confining stress-however, this extreme case will not be considered in this paper. In addition, even though the quasistatic regime has been studied in detail $[6,28]$, there is no complete picture on the structures in different flow regimes, which can offer deep insights into the underlying physics of these regimes. Only recently has the concept of structural anisotropy been added to constitutive models as it represents a microscopic measure for the deformation history (see Refs. [5,25,28], and references therein).

In the present study, we present a 3D DEM simulation to examine regime transitions in a stress-controlled model shear cell, one of the simplest practical devices in testing the rheological properties of granular materials [29-31]. The main issues we will address in this paper include: (a) the determination of regime transitions in granular flow and (b) the evolution of internal structures as the system goes through regime transitions. To the authors' knowledge, the second aspect has hardly been touched in the literature. The connection between the microscopic structure and the flow regimes will be examined as part of this study. In particular, the regime below the jamming transition, which has only recently received experimental attention in the slow quasistatic limit [32] will be studied at different shear rates. The findings can offer some insight into the dynamical characteristics for different flow regimes.

\section{SIMULATION METHOD}

\section{A. DEM approach}

In the present paper, a DEM approach [33] is used to simulate granular flow in a rectangular cell, resembling large radius annular shear cells. In such a simulation, a particle has two types of motion: translational and rotational, described by Newton's laws of motion. Since fine particles are not involved and air and moisture are not present here, forces other than mechanical contacts, e.g., particle-fluid interaction forces, can be neglected. As a result, the governing equations for the translational and rotational motions of particle $i$ with mass $m_{i}$ and moment of inertia $I_{i}$ can be written as

$$
\begin{gathered}
m_{i} \frac{d \boldsymbol{v}_{i}}{d t}=\sum_{j} \boldsymbol{F}_{i j}^{c}+\boldsymbol{F}_{i}^{g}, \\
I_{i} \frac{d \boldsymbol{\omega}_{i}}{d t}=\sum_{j} \boldsymbol{M}_{i j},
\end{gathered}
$$

where $\boldsymbol{v}_{i}$ and $\boldsymbol{\omega}_{i}$ are the translational and angular velocities of the particle, $F_{i j}^{c}$ and $\boldsymbol{M}_{i j}$ are the contact force and the torque acting on particle $i$ by particle or wall $j$, and $\boldsymbol{F}_{i}^{g}$ is the gravitational force. The contact force is composed of a (nonlinear Hertzian) elastic contact force and a damping contribution. As for the torque $\left(\boldsymbol{M}_{i j}\right)$ acting on particle $i$, a rolling friction torque is also included in addition to the torque generated by the tangential force on the particle. The rolling friction torque is generated by asymmetric normal forces and slows down the relative rotation between particles. The details on the models for contact force and torque can be seen elsewhere (e.g., Refs. [34,35]). The models used here have been successful in exploring the fundamentals of various systems (see Ref. [1] and references therein).

\section{B. Simulation conditions}

The configuration of a typical annular shear cell is shown in Fig. 1(a), which is similar to the one considered in our previous studies [36,37]. Here, to improve computational efficiency, only a flat rectangular segment of the shear cell is simulated [Fig. 1(b)], and periodic boundary conditions are used to represent the symmetrical geometry of the whole annular space. Note that the circumferential curvature is ignored in this setting. However, the key operational features of the shear cell are still maintained. This simplification has also been used by other investigators to study other aspects of annular shear cells [38,39]. The cell consists of an upper platen and a lower platen [perpendicular to the $Z$ axis and with a uniform dark color in Fig. 1(b)], two stationary walls [perpendicular to the $Y$ axis and with a dark shaded color in Fig. 1(b)], and two periodic boundary planes (perpendicular to the $X$ axis). Both the upper and the lower platens of the cell are formed by 360 glued spherical particles that have the same size and material properties as the flowing particles. This sheared granular material in the cell consists of 5000 spherical 


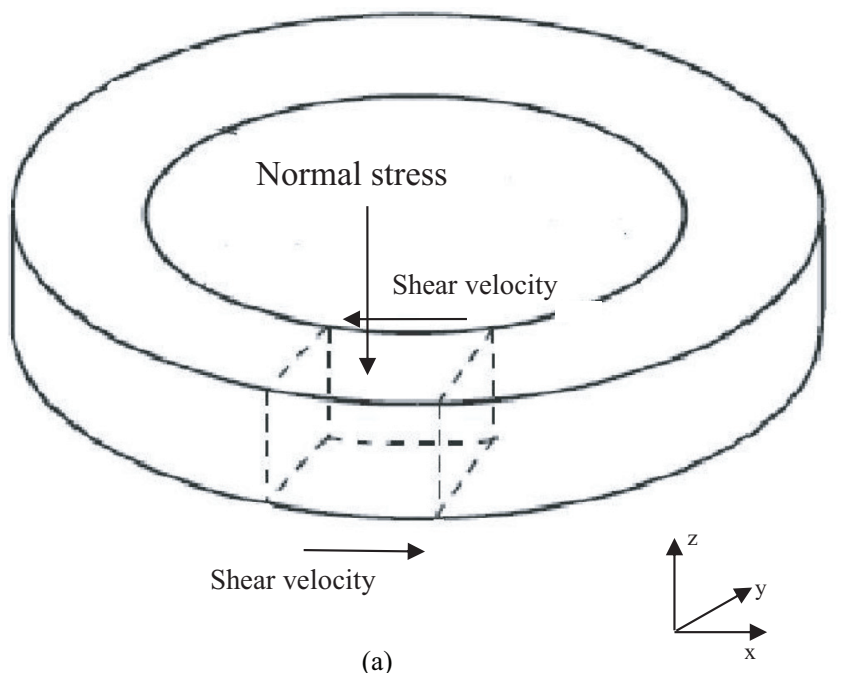

(a)

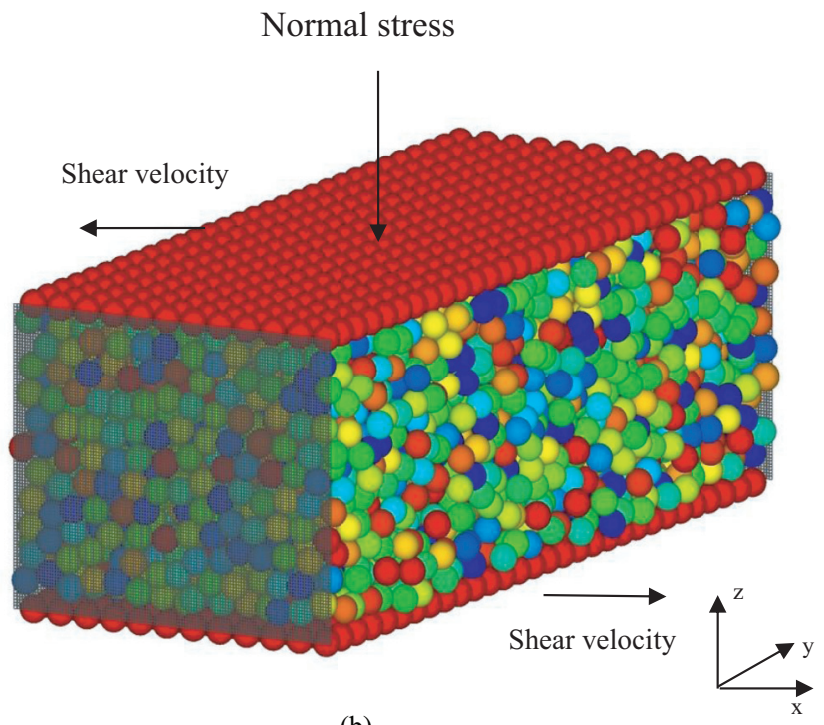

(b)

FIG. 1. (Color online) Annular shear cell geometry: (a) the section selected for simulating the shear cell under applied normal pressure and shear velocities on platens and (b) snapshot of particles under shearing between platens.

particles with a diameter of $d$. The cell dimensions are $12 d$ and $30 d$ in the $X$ and $Y$ directions, respectively, the same as those used in our previous studies [37]. The shear velocity of the platens and the normal stress applied in the negative $Z$ direction were precisely controlled. Trial tests indicate that a larger cell does not affect the results much (data not shown for brevity).

For each simulation, the 5000 spherical particles were first generated randomly and then were discharged at a preset rate from the top without the upper platen. After a certain period of time, the particles formed a packing on the lower platen. The upper platen was then placed on the top of the packing. Using a gravitational time scale as the unit of time from $t=40.0 \sqrt{d / g}$, the lower platen was given a gradually increasing shear velocity in the positive $X$ direction, and the upper platen was given both a gradually increasing shear velocity in the negative
TABLE I. Physical parameters and conditions used in the present paper.

\begin{tabular}{lcc}
\hline \hline Parameter & Value & Units \\
\hline Young's modulus $(p, w)$ & $2.5 \times 10^{6}$ & $\pi \rho d g / 6$ \\
Poisson's ratio $(p, w)$ & 0.3 & \\
Sliding friction coefficient $(p-p), \mu_{p}$ & 0.5 & \\
Sliding friction coefficient $(p-w), \mu_{w}$ & 0.3 & \\
Rolling friction $(p-p$ or $p-w), \mu_{r}$ & 0.01 & $d$ \\
Coefficient of restitution, $\varepsilon$ & 0.8 & \\
Time step & 0.0001 & $\sqrt{d / g}$ \\
Scaled stiffness, $k^{*}$ & $3 \times 10^{2}-2.5 \times 10^{5}$ & \\
Elastically scaled stress, $\sigma^{*}$ & $6 \times 10^{-6}-0.3$ & \\
Kinetically scaled stress, $\sigma^{\prime}$ & $6 \times 10^{-2}-7.5 \times 10^{4}$ & \\
\hline
\end{tabular}

Note: $d$ is the maximum particle diameter, $g\left(=9.81 \mathrm{~ms}^{-2}\right)$ is the magnitude of $\boldsymbol{g}$, and $\rho$ is the density of particles.

$X$ direction and a gradually increasing normal stress in the negative $Z$ direction. At $t=70.0 \sqrt{d / g}$, the velocities and normal stress reached the preset target values. From then, the bulk properties of the system were closely monitored. If these properties only showed minor fluctuations around constant values, then the system was assumed to be in steady state. It was observed that the shearing systems quickly became steady. Consequently, at $t=80.0 \sqrt{d / g}$, the process of calculating microstructural and dynamic properties started and was carried out until the simulations finished at $t=160.0 \sqrt{d / g}$.

Following the approach of Campbell $[17,18]$, scaled physical properties were adopted in this paper, including elastically scaled stress $\sigma^{*}=\sigma d / k$, kinetically scaled stress $\sigma^{\prime}=\sigma / \rho d^{2} \dot{\gamma}^{2}$, and scaled stiffness $k^{*}=\sigma^{\prime} / \sigma^{*}=k / \rho d^{3} \dot{\gamma}^{2}$, where $\sigma$ is the applied normal stress, $\rho$ is the particle density, $d$ is the particle diameter, $\dot{\gamma}$ is the effective shear rate due to the platens, and $k$ is the material stiffness. The physical meaning of $\sigma^{*}$ is the particle deformation induced by the applied stress, relative to its size $[17,18]$. The scaled physical parameters vary, whereas, other parameters are kept constant. The packing fraction will be used as a key parameter to study the regime transition. The simulations showed that the fluctuations in the cell height were less than $1 \%$ of its average value after the flows reached their macroscopically steady state for the cases considered. This indicates that the packing fraction has little variation in the steady state of the system. To eliminate the effect of the fluctuation, the packing fraction for each case is averaged from $t=80.0 \sqrt{d / g}$ to $t=160.0 \sqrt{d / g}$ in which the flow is considered to be in the steady state. The input and output parameters and their values are listed in Table I. The material stiffness $k$ was estimated by $k=(8 / 3) E R$, where $E$ is the effective Young's modulus, defined as $1 / E=2\left(1-\alpha^{2}\right) / E_{0}$, and $\alpha$ and $E_{0}$ are the Poisson ratio and Young's modulus of particles, respectively. $R$ is the effective radius, defined as $1 / R=2 / R_{0}$, where $R_{0}=d / 2$ is the radius of the particles [40]. Our focus in this paper is to understand the physics of the flow regimes and regime transitions from the viewpoint of structural evolution. So, the range of the scaled parameters in this paper is not as extensive as those used in the previous studies $[17,18,23,24,26]$. 


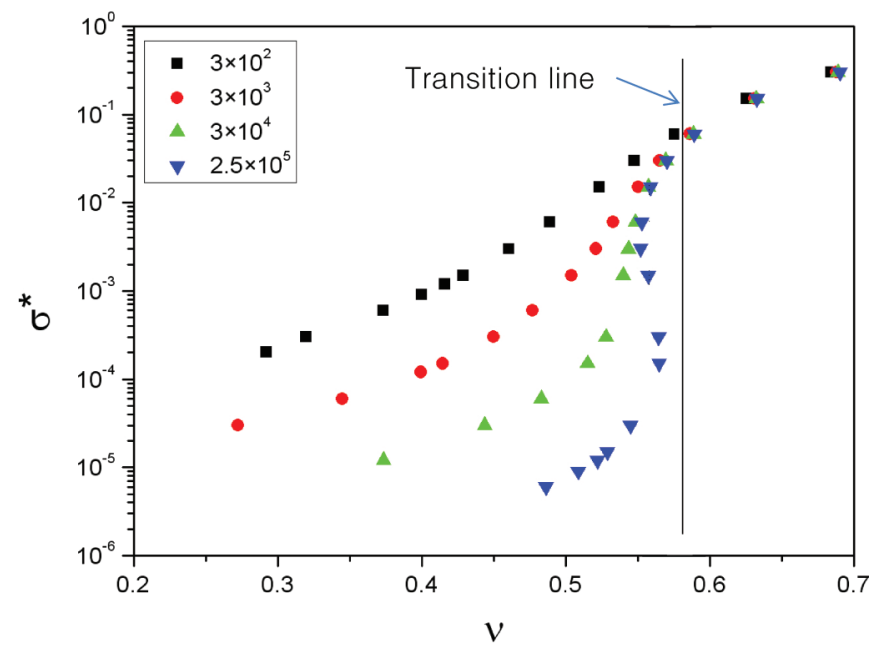

FIG. 2. (Color online) Variation in elastically scaled applied stress $\left(\sigma^{*}\right)$ with packing fraction $(v)$ for different values of scaled stiffness.

\section{RESULTS AND DISCUSSION}

In this section, we will first consider the determination of regime transitions, which are controlled by varying the values of shear rate and applied pressure. The variation in the internal structure is then examined in relation to the regime transitions. Finally, the corresponding distribution of the normal forces among particles is analyzed.

\section{A. Determination of regime transitions}

Figure 2 shows the dependence of the elastically scaled applied stress $\left(\sigma^{*}\right)$ on the packing fraction $(v)$ for different values of the scaled stiffness $\left(k^{*}\right)$. When there is no inertial effect in the system (quasistatic regime), particles are confined by their neighbors and only interact elastically. In this case, the particle deformation only depends on the applied stress. As the system constraint (applied normal stress) is relaxed, inertial effects become increasingly significant, and the particle deformation is more and more sensitive to the shear rate. Figure 2 shows that, when $v \geqslant 0.58$, the data corresponding to different values of $k^{*}$ tend to collapse into one master curve, indicating that the elastically scaled stress of the dense systems is independent of $k^{*}$ (shear rate) in the quasistatic regime. For $v<0.58$, the data for different $k^{*}$ 's start to deviate from each other, implying the appearance of inertial effects. As a result, $v=0.58$ is a critical packing fraction that separates the quasistatic and intermediate regimes, lower than 0.65 as determined in Ref. [24]. A possible reason is that, in the present paper, the existence of two stationary walls perpendicular to the $Y$ direction facilitates the formation of force chains, whereas, there are no walls in the system in Ref. [24].

In order to determine the transition between the intermediate flow and the inertial flow, we employed the same method used by Ji and Shen [24] and Aarons and Sundaresan [26]. The method involves identifying the correlation between kinetically scaled stress $\sigma^{\prime}=\sigma / \rho d^{2} \dot{\gamma}^{2}$ and packing fraction $v$. According to Campbell [18], the stresses are proportional to the product of the transported momentum and the transport

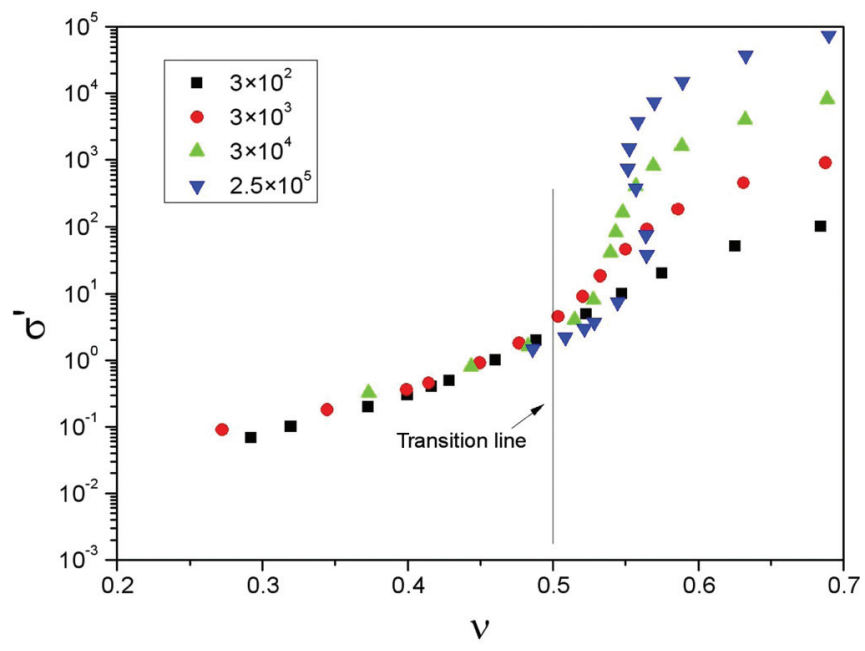

FIG. 3. (Color online) Variation in kinetically scaled applied stress $\left(\sigma^{\prime}\right)$ with packing fraction $(v)$ for different values of scaled stiffness.

rate for particles. For inertial flows, both the transported momentum and the transport rate are proportional to the shear rate. As a result, the stresses scale quadratically with the shear rate in the inertial regime so that the kinetically scaled stress $\sigma^{\prime}=\sigma / \rho d^{2} \dot{\gamma}^{2}$ can be used to identify the inertial regime. The results from this scaling are shown in Fig. 3. It is observed that the data collapse below $v=0.50$, but when the packing fraction is high (e.g., $v \geqslant 0.58$ ), the data for $\sigma^{\prime}$ for different values of $k^{*}$ demonstrate significant differences, showing that the kinetically scaled stress is greatly dependent on $k^{*}$ for shear rates in the quasistatic regime. This indicates that the stress for all the flows scales kinetically with the packing fraction below a threshold density and, thus, the flows are in the inertial regime. The same data collapse behavior and dependence of $\sigma^{*}$ and $\sigma^{\prime}$ on $v$ can also be observed in volume-controlled systems $[17,24]$. Thus, we are able to show that the rheological properties of granular flows under volume- and stress-controlled conditions are actually the same as suggested by Aarons and Sundaresan [26].

Different $k^{*}$ values were used in Figs. 2 and 3. Similar to the treatment of Aarons and Sundaresan [26], we can determine the regime transition sets of $\left(\sigma^{*}, k^{*}\right)$ and $\left(\sigma^{\prime}, k^{*}\right)$. The points close to the lines $v=0.58$ and $v=0.5$ in Figs. 2 and 3 are considered as the quasistatic to intermediate, and intermediate to inertial regime transition points, respectively. Correspondingly, $\left(\sigma^{*}, k^{*}\right)=\left(6 \times 10^{-2}, 2.5 \times 10^{5}\right), \quad\left(6 \times 10^{-2}, 3 \times 10^{4}\right)$, $\left(6 \times 10^{-2}, 3 \times 10^{3}\right), \quad$ and $\left(6 \times 10^{-2}, 3 \times 10^{2}\right), \quad$ and $\left(\sigma^{\prime}, k^{*}\right)=\left(14,841.24,2.5 \times 10^{5}\right), \quad\left(1623.16,3 \times 10^{4}\right)$, $\left(180.35,3 \times 10^{3}\right)$, and $\left(20.00,3 \times 10^{2}\right)$ were obtained for the quasistatic or intermediate regime transition, whereas, $\left(\sigma^{*}, k^{*}\right)=\left(9 \times 10^{-6}, 2.5 \times 10^{5}\right), \quad\left(1.5 \times 10^{-4}, 3 \times 10^{4}\right)$, $\left(6 \times 10^{-4}, 3 \times 10^{3}\right)$, and $\left(6 \times 10^{-3}, 3 \times 10^{2}\right)$, and $\left(\sigma^{\prime}, k^{*}\right)=$ $\left(2.226,2.5 \times 10^{5}\right), \quad\left(4.058,3 \times 10^{4}\right), \quad\left(1.804,3 \times 10^{3}\right), \quad$ and $\left(2.000,3 \times 10^{2}\right)$ are for the intermediate or inertial regime transition.

With all the transition points determined above, we are able to obtain a flow regime map. Figure 4(a) shows the regime map in the parametric space of $\left(\sigma^{*}, k^{*}\right)$. On one hand, if $k^{*}$ (shear rate) is fixed, the decrease in $\sigma^{*}$ causes the system to 


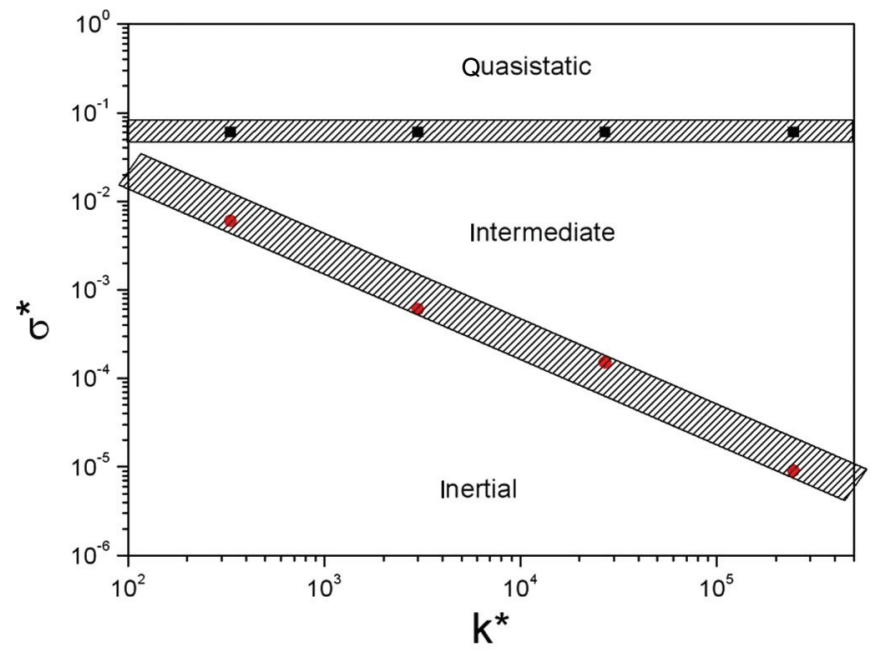

(a)

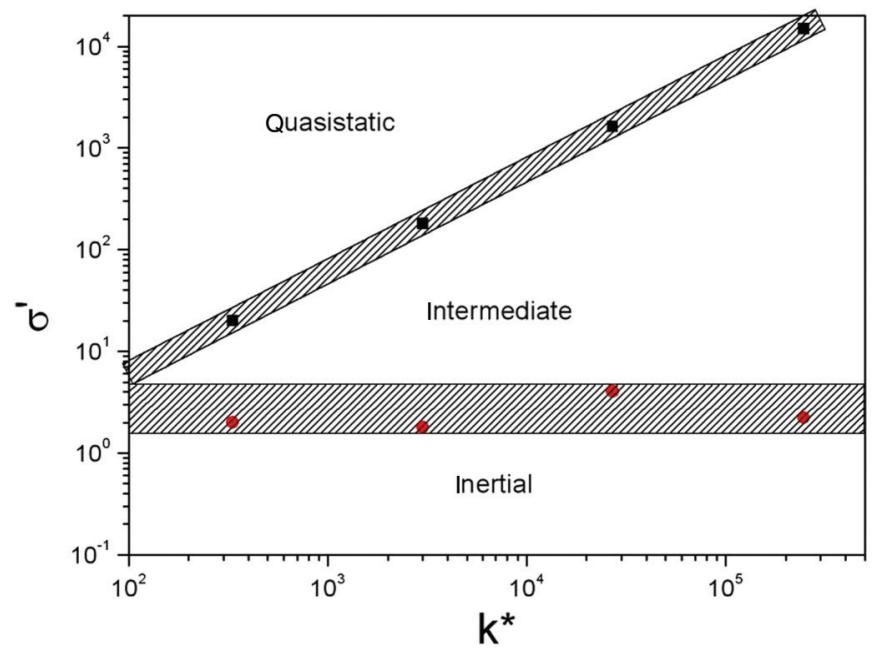

(b)

FIG. 4. (Color online) Regime map in the parametric space of: (a) $\left(\sigma^{*}, k^{*}\right)$ and (b) $\left(\sigma^{\prime}, k^{*}\right)$. The phase boundary between the quasistatic and the intermediate regimes is determined in Fig. 2. The phase boundary between the intermediate and the inertial regimes is determined in Fig. 3.

change from the quasistatic to the intermediate and, finally, to the inertial regime. This is reasonable because, for a given shear rate, decreasing the applied stress reduces the constraint on the system, allowing the system to expand. On the other hand, when $\sigma^{*}$ (applied stress) is fixed and very large, the system always stays in the quasistatic regime no matter what value the shear rate is, i.e., there is no path between quasistatic and intermediate regimes in the parametric space of $\left(\sigma^{*}, k^{*}\right)$ for fixed $\sigma^{*}$. For lower $\sigma^{*}$, the system contact constraints are mitigated so that particles can exhibit inertial effects as the shear rate becomes larger ( $k^{*}$ becomes smaller), i.e., the particles become more agitated and the system goes through the transition from the intermediate regime to the inertial regime.

We can also draw the regime map in the parametric space of $\left(\sigma^{\prime}, k^{*}\right)$ as shown in Fig. 4(b). The results in the figure indicate that the system changes from the quasistatic to the intermediate and, finally, to the inertial regime when the shear rate is fixed and the applied stress $\left(\sigma^{\prime}\right)$ is lowered. Another important feature is that, when $\sigma^{\prime}$ is low enough, the system always stays in the inertial regime. This is understandable since $\sigma^{\prime}$ by definition is the ratio of elastic stress (force chain intensity, as discussed below) and kinetic energy in the system. A small value of $\sigma^{\prime}$ indicates that particles in the system are highly agitated, which, naturally, is the characteristic of the inertial regime. As $\sigma^{\prime}$ becomes larger, large scale force chains become increasingly predominant. In this case, for a fixed $\sigma^{\prime}$, the decrease in $k^{*}$ (or the increase in shear rate) will make the system transit from the intermediate to the quasistatic regime due to the increased applied pressure (or particle deformation). Note that the developing trend of the borderlines between two regimes would change if $k^{*}$ is out of the range considered in Fig. 4. More detailed information can be seen in Ref. [18]. Furthermore, only one of the two regime plots is unique since they are connected via the definition of $k^{*}=\sigma^{\prime} / \sigma^{*}$.

We have also considered the effect of particle material properties, including sliding friction, rolling friction, and the normal restitution coefficient on the features considered above. These properties do not affect the qualitative trends of $\sigma^{*}$ and $\sigma^{\prime}$ with $v$ much, but they have an influence on the values of the critical packing fraction for the regime transitions. For example, an increasing sliding friction coefficient decreases both critical packing fractions for the quasistatic to intermediate, and intermediate to inertial regime transitions. Increasing rolling friction lowers the value of $v$ for the transition into the quasistatic regime, whereas, the increase in restitution coefficient boosts the value for the intermediate or inertial regime transition. Such issues will be discussed in more detail in another paper.

\section{B. Structural analysis}

In order to qualitatively examine the structure of the studied shear flows, we first look at the contact networks under different conditions of $\left(\sigma^{*}, k^{*}\right)$. It should be noted that, as the variation in the magnitude of normal force is huge for different cases in the present paper, it is inconvenient to compare the normal force networks using a universal scaling (typical examples of normal force networks can be found in Refs. [28,41-43]). As a consequence, we plot the networks of scaled normal forces $(f)$, which is defined as the ratio of normal contact forces $\left(F_{n}\right)$ to the average normal contact force $\left(\left\langle F_{n}\right\rangle\right)$ for a specific case, i.e., $f=F_{n} /\left\langle F_{n}\right\rangle$. In this way, we can compare the network or connectivity profiles of different cases using the same scale. In the contact network diagrams of the present paper, each stick represents a line joining the centers of two contacting particles, and its thickness stands for the magnitude of the scaled normal contact force between them. To be illustrative and to avoid artifacts in the vicinity of the walls, the figures are constructed based on the particles in the central slice ( $2 d$ in thickness) along the shear ( $x$-axis) direction.

Figure 5 shows the contact force networks for $k^{*}=$ $3 \times 10^{2}$ and eight different values of $\sigma^{*}$ together with the corresponding packing fractions and average coordination numbers. The average coordination number is the bulk average for all the sheared particles in the system. In Fig. 5(a), $\sigma^{*}$ 


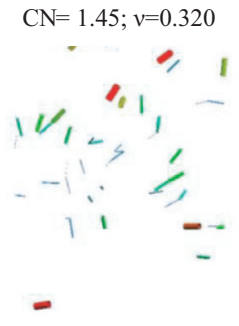

(a)

$\mathrm{CN}=4.79 ; v=0.548$

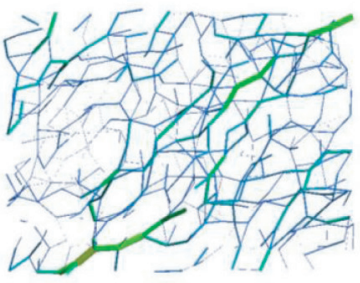

(e)

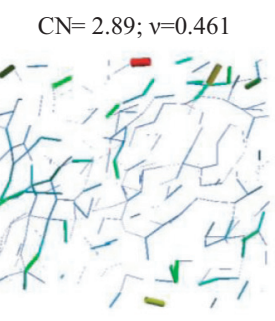

(b)

$\mathrm{CN}=5.37 ; \mathrm{v}=0.575$

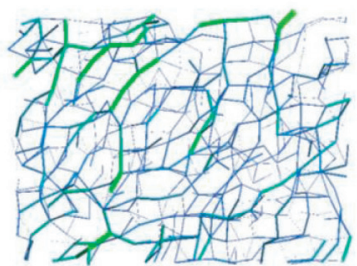

(f)

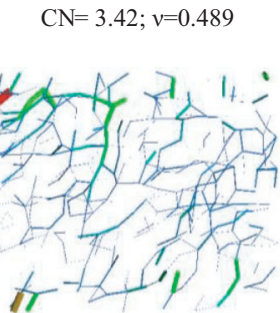

(c)

$\mathrm{CN}=6.14 ; v=0.625$

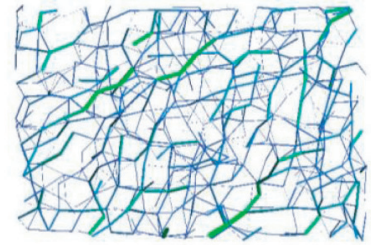

(g)
$\mathrm{CN}=4.20 ; v=0.523$

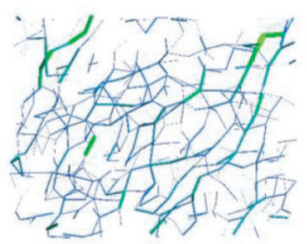

(d)

$\mathrm{CN}=6.81 ; v=0.684$

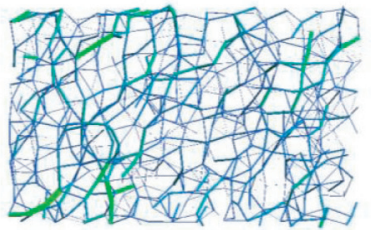

(h)

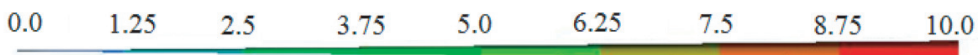

FIG. 5. (Color online) Networks of scaled forces $\left(f=F_{n} /\left\langle F_{n}\right\rangle\right)$ and the corresponding average coordination number and packing fraction when $k^{*}=3 \times 10^{2}$ for different $\sigma^{*}$ 's: (a) $3 \times 10^{-4}$, (b) $3 \times 10^{-3}$, (c) $6 \times 10^{-3}$, (d) $1.5 \times 10^{-2}$, (e) $3 \times 10^{-2}$, (f) $6 \times 10^{-2}$, (g) 0.15 , and (h) 0.3 .

takes a relatively low value of $3 \times 10^{-4}$, and the system is in the inertial regime, i.e., relatively dilute with packing fraction 0.32 . An average coordination number of 1.45 means that multiparticle contacts are infrequent and connectivity among particles is very low. Scaled contact forces of both high and low magnitudes exist in the system, implying the high heterogeneity of the force network in the inertial regime. As $\sigma^{*}$ increases to $6 \times 10^{-3}$ [Fig. 5 (c)] via the value of $3 \times 10^{-3}$ [Fig. 5(b)], the system goes through the inertial regime and reaches the boundary between the inertial and the intermediate regimes. The connectivity among particles increases as manifested by the increase in the coordination number from 1.45 to 3.42 due to the increasing system constraint and the resultant growth in the packing fraction. It is also noticed that there is an increasing number of weak scaled contact forces in the background, implying that their percentage increases in the process, whereas, that of the strong scaled forces is diminishing. The system enters the intermediate regime as $\sigma^{*}$ increases beyond $6 \times 10^{-3}$, for example, to $1.5 \times 10^{-2}$ [Fig. 5(d)] and $3 \times 10^{-2}$ [Fig. 5(e)]. The characteristic of this stage is that the internal structure becomes more and more closely knit, which is manifested by the steady increase in the average coordination number from 3.42 to 4.79 . In addition, both the relatively infrequent appearance of the large scaled forces and the stronger background of the weak forces indicate that the system considered becomes more dense and homogeneous. After $\sigma^{*}$ increases above the boundary value of $6 \times 10^{-2}$ [Fig. 5(f)], the system starts to enter the quasistatic regime. Figures $5(\mathrm{~g})$ and $5(\mathrm{~h})$ demonstrate the contact networks when $\sigma^{*}=0.15$ and 0.3 , respectively. Note that, in the quasistatic regime, there seems to be little visible variation in the dense internal structure despite an increase in connectivity, implying the high stability of the internal structure of quasistatic flows. For a more quantitative analysis of the contact network under different densities in the inertial and quasistatic regimes, see Refs. [25,44] and references therein.

We compare our results with the contact force networks of a simple shear flow in different flow regimes reported in Ref. [18] and find that the agreement is apparent, despite the fact that the contact forces in Ref. [18] are not scaled by their average in each case. The networks shown in Figs. 5(a), 5(d), and 5(e) in the present paper are consistent with the collisional and elastic-inertial networks reported in Figs. 2(d), 2(c), and 2(b) in Ref. [18], respectively. The quasistatic networks shown in Figs. 5(g) and 5(h) in this paper also agree well with their counterpart in Fig. 2(a) in Ref. [18]. This agreement implies that there are some universal features of granular flows in the same flow regime, even if the flows have different configurations or boundary conditions.

The research on force chains has been a very important component in the demarcation and characterization of different flow regimes (see Ref. [45], for example). It has been reported that the parts where there are no force chains can be associated with the existence of inertial effects [46]. In this paper, we examine the characteristics of force chains (if any) for different flow regimes for the considered systems. To this end, following the work of Peters et al. [45], we define a force chain as a quasilinear particle assembly where stress is concentrated; concentrated stress means that the contact forces 


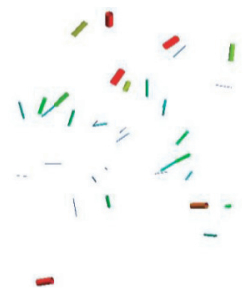

(a)

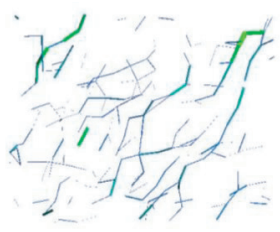

(c)

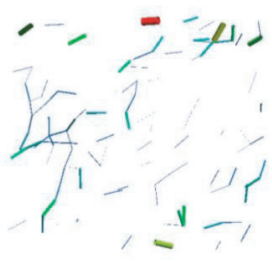

(b)

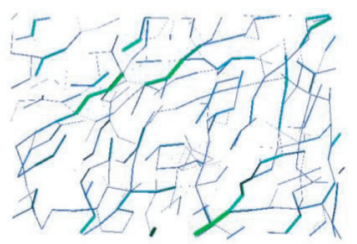

(d)

\begin{tabular}{lllllllll}
1.0 & 2.13 & 3.25 & 4.38 & 5.5 & 6.63 & 7.75 & 8.88 & 10.0 \\
\hline
\end{tabular}

FIG. 6. (Color online) Networks of large scaled forces $(f>1)$ and the corresponding average coordination number and packing fraction when $k^{*}=3 \times 10^{2}$ for different $\sigma^{*}$ s: (a) $3 \times 10^{-4}$, (b) $3 \times 10^{-3}$, (c) $1.5 \times 10^{-2}$, and (d) 0.15 . The scale of all subfigures is shown at the bottom.

with magnitudes greater than the average form connected force chains. Therefore, we remove, from Fig. 5, the scaled contact forces $(f)$ with magnitudes less than or equal to one where the cutoff is an arbitrary but convenient or simple choice. The remaining scaled forces satisfy the condition of $f>1$, i.e., $\left.F_{n}\right\rangle\left\langle F_{n}\right\rangle$. For brevity, only four cases corresponding to Figs. 5(a), 5(b), 5(d), and 5(e) are plotted in Fig. 6. Note that the quantitative examination of the quasilinear and particle assembly conditions is beyond the scope of this paper, but we can provide some qualitative descriptions here.

Figures 6(a) and 6(b) depict the structure of large scaled forces $(f>1)$ in the inertial regime. The difference is that the case in Fig. 6(b) is closer to the boundary between the inertial and the intermediate regimes. The main interaction mode in Fig. 6(a) is binary collisions, with no strong concentrated force chains formed in the system. This clearly corresponds to the traditional rapid flow or the inertial-collisional regime reported in Refs. [17-19]. In Fig. 6(b), the particle interactions become more frequent than those in Fig. 6(a). Some local contact clusters or chains appear, implying that the flow could be in the so-called inertial-noncollisional regime reported in Refs. [17-19]. According to Campbell [17-19], particles should break free from force chains in the inertial regime so there are no significant long force chains in this regime. However, we find that there are some (temporary) shortrange force chains with three to four stress-bearing particles in the inertial regime in Fig. 6(b). Therefore, the inertialnoncollisional regime may include not only particle clusters, but also short force chains for some cases. In Fig. 6(c), one can find that system-spanning force chains start to form in the intermediate regime with a respective increase in connectivity and the packing fraction. Considering the force chains are short in the inertial flow, we may conclude that the appearance of system-spanning force chains, instead of the short force chains in granular flows with high shear rates, can be used as an indication of the emergence of quasistatic effects. As $\sigma^{*}$ increases further, the number of the system-spanning force chains grows, and inertial domains (i.e., no force chains) are gradually "squeezed" out of the system. When the inertial effects disappear with the application of higher normal stresses, the system enters the quasistatic regime as shown in Fig. 6(d), which is characterized by the percolation of system-spanning force chains through the whole cell [32].

At this point, we should remark that Campbell [18] proposed a different method to determine the quasistatic to intermediate regime transition points. According to his method, the points where the data for different $k^{*}$ 's deviate from the critical state (or transition) are considered as the indicators of the quasistatic to intermediate regime transition. This method is not suitable for some cases considered here as demonstrated in the examination of the relevant force chains. Figure 6(c) belongs to the intermediate regime according to the flow chart drawn in Fig. 4(a), and it is in the low stress critical state [18] as shown in Fig. 2. According to the criterion in Ref. [18], Fig. 6(c) would belong to the quasistatic regime since its corresponding point in the $\left(\nu, \sigma^{*}\right)$ space in Fig. 2 does not deviate from the critical state line. However, the strong scaled force network in Fig. 6(c) clearly shows that the system-spanning force chains have not percolated the whole system yet and some part of the cell space is still dominated by inertial domains (with no force chains formed), implying that the flow is in the intermediate regime.

\section{Force statistics}

The force network among particles in a static or dynamic state can be analyzed in terms of the force probability distribution, which can give us some hints regarding the general behavior of granular systems [28,47]. Many studies have been devoted to determining the shape of the probability density distribution of the normal contact forces between particles $P(f)$ and its variation under different conditions [48-51] in addition to the long-range correlations in the forces [28]. Efforts have also been made to investigate the signature change in $P(f)$ when systems go through jamming transitions [51-53]. In this paper, we use similar ideas to determine if there is any change in the shape of $P(f)$ as the granular flow changes between different flow regimes. Note that, due to shear and the consequent anisotropy, the orientation angle of the contact forces is important for the analysis of two-dimensional force distributions (see Ref. [54] and references therein). This complicated issue in three dimensions will be considered in our future study.

The probability distributions of the scaled normal contact force $f\left(=F_{n} /\left\langle F_{n}\right\rangle\right)$ for flows under different conditions are shown in Fig. 7. In general, the distribution becomes wider as the flow goes through the quasistatic, intermediate, or inertial regime transitions. In the quasistatic regime, the force distributions obtained in the present paper have a similar trend to those for static packings obtained in Refs. [28,48,49]. The 


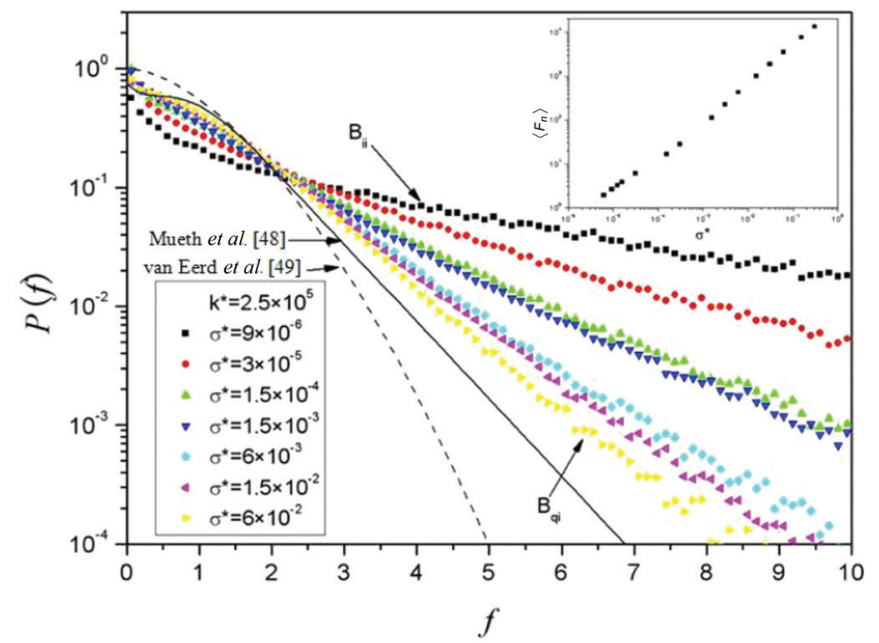

(a)

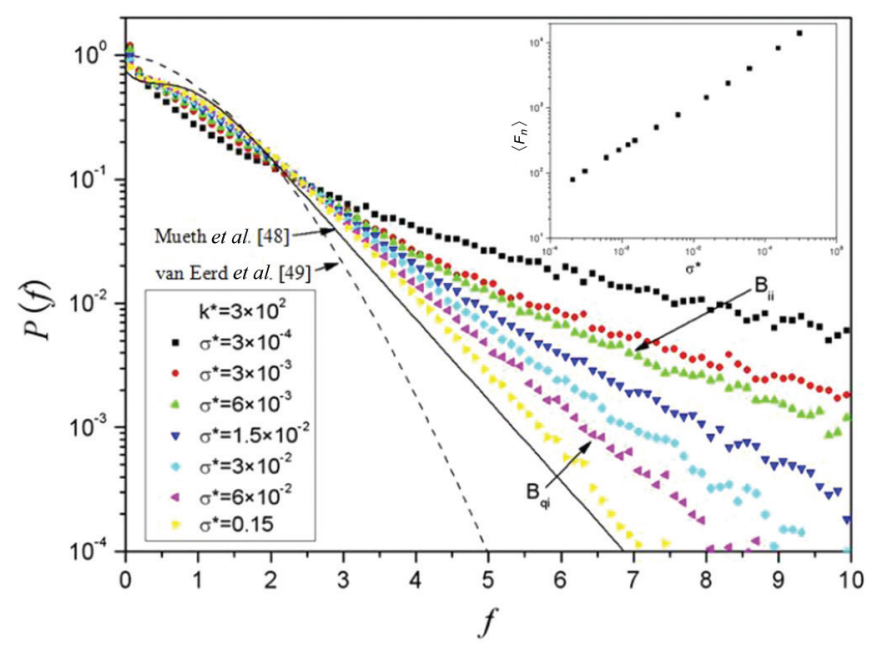

(b)

FIG. 7. (Color online) Probability density distributions of the scaled normal forces $\left(f=F_{n} /\left\langle F_{n}\right\rangle\right)$ for different $\sigma^{*}$ 's when: (a) $k^{*}=2.5 \times 10^{5}$ and (b) $k^{*}=3 \times 10^{2}$. Quasistatic to intermediate, and intermediate to inertial phase boundaries are marked as $B_{\mathrm{qi}}$ and $B_{\mathrm{ii}}$, respectively. The solid and dashed lines stand for the force distributions obtained by Mueth et al. [48] and van Eerd et al. [49], respectively. The insets show the variation in the average contact force $\left(\left\langle F_{n}\right\rangle\right)$ with $\sigma^{*}$.

force chains percolate the whole system, and the majority of particles contribute to the network bearing large forces as, for example, shown in Fig. 6(d). At the same time, the average normal force $\left\langle F_{n}\right\rangle$ is large (see the insets of Fig. 7 for the variation in $\left\langle F_{n}\right\rangle$ ), and the distribution is relatively even. In the intermediate and inertial regimes, the force chains gradually disappear, and an increasing number of particles interact by collisions. The examples of the force networks in the inertial regime can be seen in Figs. 6(a) and 6(b). Only a limited number of collisions are noticeable, and most interactions are fairly weak, resulting in a very small $\left\langle F_{n}\right\rangle$ (the insets of Fig. 7). As a result, the corresponding scaled force distribution is rather wide.

In Fig. 7, we include the force distributions corresponding to the boundary points of the quasistatic to intermediate $\left(B_{\mathrm{qi}}\right)$

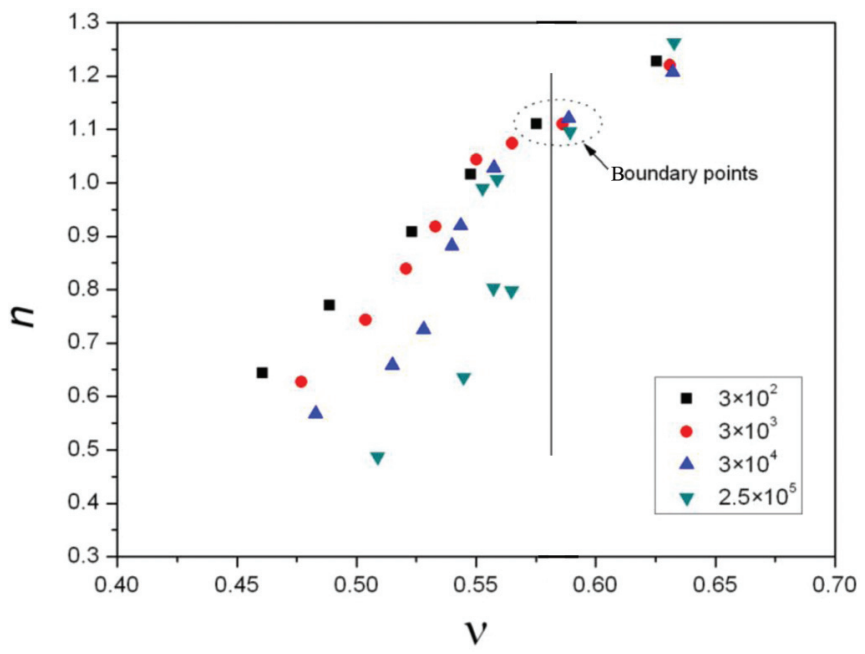

FIG. 8. (Color online) Variation in the power index $n$ in Eq. (3) with packing fraction $v$ for different scaled stiffnesses. Note that all the points on the quasistatic to intermediate regime boundary (highlighted as boundary points) have $n$ values close to 1.1 .

and intermediate to inertial $\left(B_{\mathrm{ii}}\right)$ transitions. Although the distributions vary for $B_{\mathrm{ii}}$ under different values of $k^{*}$, they remain invariant for $B_{\mathrm{qi}}$. This is confirmed for many other values of $k^{*}$ (data not shown for brevity). That is, the force distributions of the granular flows under different shear rates share a common feature when they go through the quasistatic to intermediate transition. The tails (large $f$ range) of the force distributions can be fitted by a stretched exponential,

$$
P(f)=\exp \left(-c f^{n}\right),
$$

where $c$ and $n$ are fitting parameters. This functional form is consistent with the one in Refs. $[28,49]$. In this paper, the best-fitting values of $n$ close to the inertial to intermediate and intermediate to quasistatic regime boundaries are examined in terms of its correlation with $v$ as shown in Fig. 8. The best fitting to the force distributions of $B_{\mathrm{qi}}$ under different $k^{*}$ 's corresponds to $n=1.1$ with a typical variation or uncertainty of 0.05 . Using $n=1.1$ as a demarcation point, $n>$ 1.1 is for a flow in the quasistatic regime, and a higher value of $n$ means the flow is deeper into the quasistatic regime; $n<1.1$ corresponds to a flow in the intermediate to inertial regime, and a lower $n$ signifies larger inertial effects. We note that this is a remarkable common feature in the force distributions for all the considered flows at different $k^{*}$ 's, and one can tell if there is any significant inertial component in a granular flow from the tail of its force distribution. Therefore, the value of $n$ can potentially serve as an alternative indicator for the emergence of the quasistatic regime.

\section{Coordination number in regime transitions}

In this section, we try to quantitatively explain why the demarcation between the quasistatic and the intermediate regimes works from the microdynamic perspective. Figure 9(a) shows the variation in the $\mathrm{CN}$ with the packing fraction $(v)$ for different scaled stiffnesses $k^{*}$. The data can be categorized into two groups, divided by a demarcation point corresponding to $v=0.58$ with a critical coordination number of around 5.6 


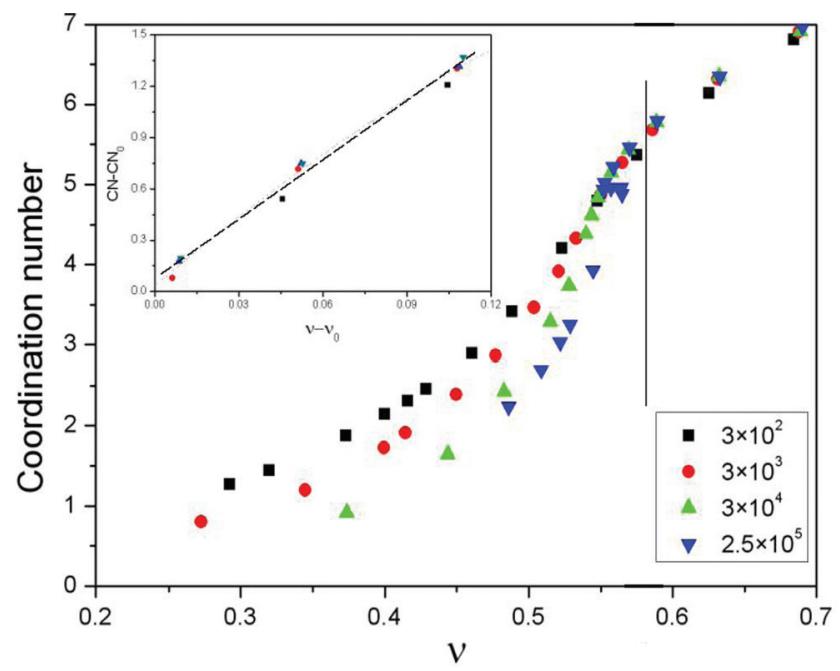

(a)

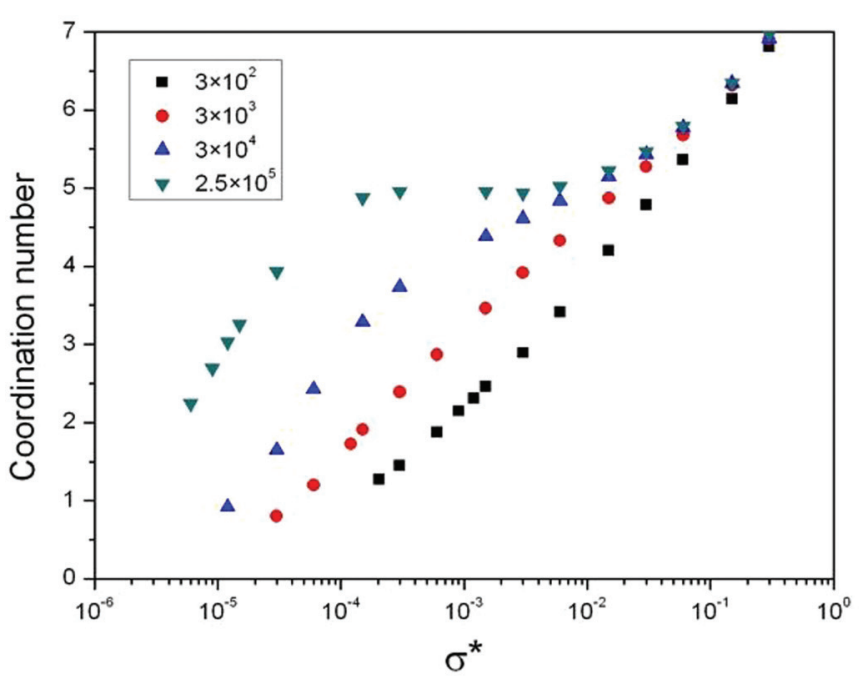

(b)

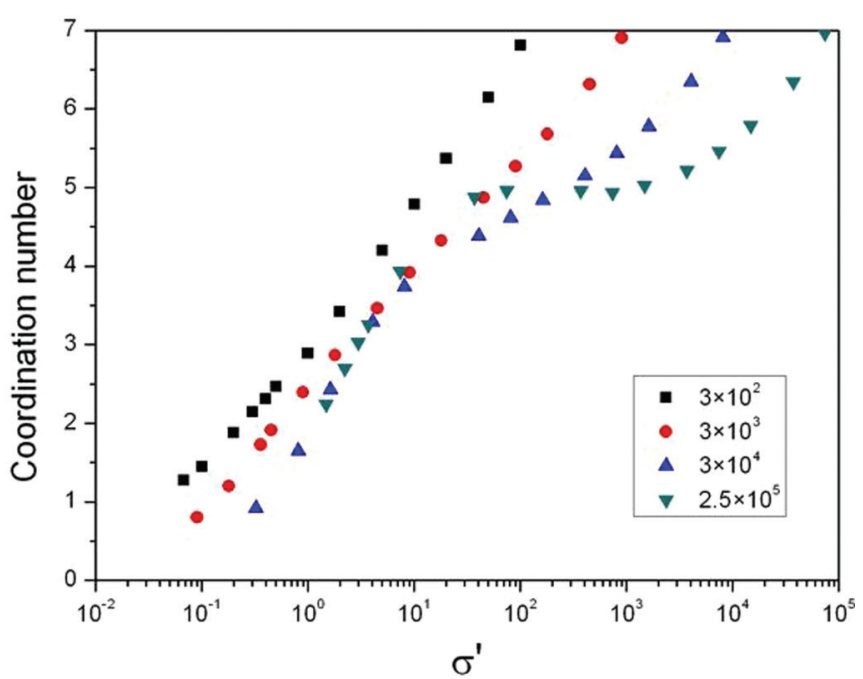

(c)

FIG. 9. (Color online) Variation in coordination number with: (a) packing fraction $v$, (b) elastically scaled applied stress $\sigma^{*}$, and (c) kinetically scaled applied stress $\sigma^{\prime}$ for different scaled stiffnesses. The vertical line in the main panel of (a) corresponds to the critical packing fraction of 0.58 . The inset shows the scaling between $\mathrm{CN}-\mathrm{CN}_{0}$ and $v-v_{0}$ with the dotted curve standing for the best fit given in Sec. III D.

(which is close to the isostatic point 6.0). In the first group where $v<0.58$, the $\mathrm{CN}$ increases with the decrease in $k^{*}$ (or the increase in shear rate) for a given $v$; whereas, in the second group where $v>0.58$, the variation in the $\mathrm{CN}$ with $v$ is independent of $k^{*}$, and the data of the $\mathrm{CN}$ collapse more or less. The first group belongs to the flow regimes in which inertial effects play an obvious role and any increase in shear rate enhances particle contacts; the flow characteristics are, hence, influenced by the shear rate (i.e., $k^{*}$ ). As a result, the data in the first group should be in the intermediate or inertial regimes. For the second data group, the fact that the increase in shear rate cannot cause any change in particle contacts indicates that the force chains have percolated the whole system. Naturally, the corresponding systems are practically rate independent and, thus, belong to the quasistatic regime.

In Sec. III A, we showed that the data of $\sigma^{*}$ collapse when $v$ exceeds the same critical packing fraction (0.58). As a result, one would expect that there should also be a data collapse between the $\mathrm{CN}$ and the $\sigma^{*}$ when the $\mathrm{CN}$ is higher than its critical value $(\sim 5.6)$. To test this speculation, we plot the correlation between the $\mathrm{CN}$ and the $\sigma^{*}$ for different $k^{*}$ 's in Fig. 9(b). As expected, the data pattern presented in this figure is highly similar to that in Fig. 2, and the data for the $\mathrm{CN}$ and $\sigma^{*}$ collapse when the $\mathrm{CN}$ exceeds $\sim 5.6$. Therefore, it is also feasible to use the correlation between the $\mathrm{CN}$ and the $\sigma^{*}$ to determine the intermediate or quasistatic regime boundary. For the correlation between the $\mathrm{CN}$ and the $\sigma^{\prime}$, there is no such collapse as indicated in Fig. 9(c) neither for the inertial nor for the quasistatic regime.

In Sec. III B, we showed that, as the flows enter the quasistatic regime, the force chains percolate through the whole system and all the particles in the system are tightly confined in the contact network as observed in Figs. 5(g)-5(h) and 6(d). This phenomenon is reminiscent of global jamming 
in granular packings [55] and strongly resembles the observations of jamming under shear [32]. The packing fraction (0.55), corresponding to the onset of global jamming [55], appears close to the one $(0.58)$ for the quasistatic to intermediate transition in the present study. However, changes in packing fractions as small as 0.03 can lead to enormous changes in the $\mathrm{CN}$ and even more in $\sigma^{*}$ so that one has to be careful when defining differences in jamming packing fractions $[25,28]$. It would be of interest to examine further if and how global jamming is truly related to this regime transition.

The concept of jamming has long been used to describe the transition to rigidity of a series of disordered materials, such as foams, granular matter, and glasses [56,57]. The behavior of materials near and above the jamming transition is extensively studied with regard to the correlation between the average coordination number and the packing fraction, whereas, much less attention has been given to the regime below jamming [32]. It is found that, both experimentally and numerically, a granular system going through a global jamming transition exhibits the following two attributes [58-63]: (a) The average coordination number $\mathrm{CN}$ of the system increases sharply at the transition point when increasing the packing fraction $v$ above a certain value $v_{0}$ (see Ref. [28] and references therein), and (b) the $\mathrm{CN}$ still increases as a function of $\Delta v\left(\Delta v=v-v_{0}\right)$ above $v_{0}$ with the transition point defined as $\left(v_{0}, \mathrm{CN}_{0}\right)$. Both attributes are suspect to finite size effects and have to be considered very carefully.

For frictionless spheres [28,58-61], a simple scaling feature exists above the jamming transition point in the form of $\mathrm{CN}-\mathrm{CN}_{0}=\left(v-v_{0}\right)^{\beta}$, where $\beta \sim 0.5$, whereas, for frictional particles, different values of $\nu_{0}$ and $\mathrm{CN}_{0}$ are identified with very similar values of $\beta$. More recently, Imole et al. [25] reported that the jamming transition packing fraction is also a function of the applied deformation mode, but they also confirmed that the scaling holds almost perfectly, whereas, it is independent of force laws [62]. To check the scaling law in the present sheared system, we plot $\mathrm{CN}-\mathrm{CN}_{0}$ against $v-v_{0}$ in the inset of Fig. 9(a) $\left(v_{0}\right.$ and $\mathrm{CN}_{0}$ are 0.58 and 5.6 , respectively). We can see that the data collapse (except for the smallest $k^{*}$ ), indicating that the flow becomes globally jammed when $v$ is larger than 0.58 , is the demarcation point for the quasistatic to intermediate transition. We can get the best fit for the data points as $\Delta \mathrm{CN}=14.021 \Delta v-21.007(\Delta v)^{2}$, where $\Delta \mathrm{CN}=\mathrm{CN}-\mathrm{CN}_{0}$ and $\Delta v=v-v_{0}$ with a correlation coefficient of 0.992 . Thus, the correlation between $\mathrm{CN}-\mathrm{CN}_{0}$ and $v-v_{0}$ takes a quadratic form in the present granular flows instead of the square-root form found in the static granular packing [58-62]. This may be attributed to the fact that, although the structural change in the present system can be analyzed in terms of global jamming transition, dynamic granular flow may be very different from static granular packings.

\section{CONCLUSIONS}

The shearing of particles in a model shear cell under stresscontrolled conditions has been investigated numerically using the discrete element method. Granular flows under different conditions of scaled stiffness $\left(k^{*}=k / \rho d^{3} \dot{\gamma}^{2}\right)$ and scaled normal stress $\left(\sigma^{*}=\sigma d / k\right)$ are considered, and the packing fraction ( $\nu)$ and kinetically scaled stress $\left(\sigma^{\prime}=\sigma / \rho d^{2} \dot{\gamma}^{2}\right)$ for each flow are calculated. The data collapse between $\sigma^{*}$ and $v$ for different $k^{*}$ 's when $v>0.58$ is considered as the lower limit packing fraction of the quasistatic regime, whereas, the data collapse between $\sigma^{\prime}$ and $v$ for different $k^{*}$ 's when $v<0.50$ is deemed as the indication (upper limit) of the inertial regime. This regime demarcation method of identifying two critical packing fractions is consistent with that used in Ref. [24] but is different from that using the low stress critical state [18].

The internal structures of the flows in different regimes (quasistatic, intermediate, or inertial) have been investigated concerning the evolution of contact networks and force chains for different combinations of $\sigma^{*}$ and $k^{*}$. The results show that the different attributes of the three flow regimes and the corresponding transitional behaviors can adequately be reflected in the contact networks or force chains together with the information about the average coordination number and packing fraction. A striking result found here is that, contrary to common belief, short-range force chains are observed in the inertial regime for large shear rates. The implication of this finding for the inertial (transitional) regime is that such a flow may contain not only particle clusters as shown by Campbell in Ref. [18], but also short or localized force chains. Furthermore, the percolation of system-spanning force chains is a characteristic of the quasistatic regime.

The force statistics analysis shows that the force distribution becomes increasingly wide as the flow transits from the quasistatic to the inertial regime, a phenomenon also observed in thermal systems when temperature increases. The tail of the probability density distribution of scaled normal forces can be well approximated by the form $P(f)=\exp \left(-c f^{n}\right)$. The power index $n$ may serve as a new flow regime indicator with $n>$ 1.1 for the quasistatic regime and $n<1.1$ for the intermediate and inertial regimes. This critical $n$ may vary with material properties, which should be studied in the future.

The correlation between the $\mathrm{CN}$ and the packing fraction $v$ is established to testify the robustness of the present demarcation method. It is found that the dependence of the $\mathrm{CN}$ on $\sigma^{*}$ can also be used to identify the quasistatic to intermediate regime transition since the same data collapse happens as that between $\mathrm{CN}$ and $v$. By examining the correlation between $\mathrm{CN}$ and $v$, we confirm, as observed earlier in quasistatic situations [25,60], that the transition to the quasistatic regime is related to the global jamming transition. A square-root scaling between $\Delta \mathrm{CN}\left(=\mathrm{CN}-\mathrm{CN}_{0}\right)$ and $\Delta v\left(=v-v_{0}\right)$ also exists for the present sheared system. This result indicates that, although granular shear flows can differ from granular packings in many ways, the snapshots and the averaged (isotropic) properties of their internal structures are comparable. They may share some common characteristics, which should be explored further in future studies, especially concerning anisotropy [25].

\section{ACKNOWLEDGMENTS}

The authors are grateful to the Australian Research Council and the University of New South Wales for the financial support of the present paper. S.L. especially appreciates a visiting fellowship award by UNSW for his research visit. 
[1] H. P. Zhu, Z. Y. Zhou, R. Y. Yang, and A. B. Yu, Chem. Eng. Sci. 63, 5728 (2008).

[2] Anonymous, Science 309, 78 (2005).

[3] A. Schofield and C. P. Wroth, Critical State in Soil Mechanics (McGraw-Hill, New York, 1968).

[4] R. M. Nedderman, Statics and Kinematics of Granular Materials (Cambridge University Press, Cambridge, UK, 1992).

[5] S. Luding, J. Phys.: Condens. Matter 17, S2623 (2005).

[6] S. Luding and F. Alonso-Marroquin, Granular Matter 13, 109 (2011).

[7] S. B. Savage and D. J. Jeffrey, J. Fluid Mech. 110, 255 (1981).

[8] J. T. Jenkins and S. B. Savage, J. Fluid Mech. 130, 187 (1983).

[9] C. K. K. Lun, S. B. Savage, D. J. Jeffrey, and N. Chepurniy, J. Fluid Mech. 140, 223 (1984).

[10] C. K. K. Lun and S. B. Savage, Acta Mech. 63, 15 (1986).

[11] C. S. Campbell, J. Fluid Mech. 203, 449 (1989).

[12] C. K. K. Lun, J. Fluid Mech. 233, 539 (1991).

[13] J. T. Jenkins and D. Berzi, Granular Matter 14, 79 (2012).

[14] GDR MiDi, Eur. Phys. J. E 14, 341 (2004).

[15] O. Pouliquen and F. Chevoir, C. R. Phys. 3, 163 (2002).

[16] G. I. Tardos, S. McNamara, and I. Talu, Powder Technol. 131, 23 (2003).

[17] C. S. Campbell, J. Fluid Mech. 465, 261 (2002).

[18] C. S. Campbell, J. Fluid Mech. 539, 273 (2005).

[19] C. S. Campbell, Powder Technol. 162, 208 (2006).

[20] M. Massoudi and T. X. Phuoc, Powder Technol. 175, 146 (2007)

[21] Y. Forterre and O. Pouliquen, Annu. Rev. Fluid Mech. 40, 1 (2008).

[22] M. Babic, H. H. Shen, and H. T. Shen, J. Fluid Mech. 219, 81 (1990).

[23] L. Aarons and S. Sundaresan, Powder Technol. 169, 10 (2006).

[24] S. Y. Ji and H. H. Shen, J. Rheol. 52, 87 (2008).

[25] O. I. Imole, N. Kumar, V. Magnanimo, and S. Luding, KONA 30, 84 (2013).

[26] L. Aarons and S. Sundaresan, Powder Technol. 183, 340 (2008).

[27] C. S. Campbell, Phys. Fluids 23, 013306 (2011).

[28] M. K. Müller, T. Pöschel, and S. Luding, Chem. Phys. 375, 600 (2010).

[29] S. B. Savage and M. Sayed, J. Fluid Mech. 142, 391 (1984).

[30] D. M. Hanes and D. L. Inman, J. Fluid Mech. 150, 357 (1985).

[31] S. S. Hsiau and W. L. Yang, Phys. Fluids 14, 612 (2002).

[32] D. Bi, J. Zhang, B. Chakraborty, and R. P. Behringer, Nature (London) 480, 355 (2011).

[33] P. A. Cundall and O. D. L. Strack, Geotechnique 29, 47 (1979).

[34] H. P. Zhu, Z. Y. Zhou, R. Y. Yang, and A. B. Yu, Chem. Eng. Sci. 62, 3378 (2007).

[35] H. P. Zhu and A. B. Yu, Physica A 325, 347 (2003).

[36] X. Wang, H. P. Zhu, and A. B. Yu, Granular Matter 14, 411 (2012).
[37] X. Wang, H. P. Zhu, and A. B. Yu, Phys. Fluids 24, 053301 (2012).

[38] A. Hassanpour, Y. Ding, and M. Ghadiri, Adv. Powder Technol. 15, 687 (2004).

[39] Z. Ning and M. Ghadiri, Chem. Eng. Sci. 61, 5991 (2006).

[40] H. Hertz, J. Reine Angew. Math. 92, 156 (1882).

[41] C.-h. Liu, S. R. Nagel, D. A. Schecter, S. N. Coppersmith, S. Majumdar, O. Narayan, and T. A. Witten, Science 269, 513 (1995).

[42] D. W. Howell, R. P. Behringer, and C. T. Veje, Phys. Rev. Lett. 82, 5241 (1999).

[43] D. W. Howell, R. P. Behringer, and C. T. Veje, Chaos 9, 559 (1999).

[44] J. Sun and S. Sundaresan, J. Fluid Mech. 682, 590 (2011).

[45] J. F. Peters, M. Muthuswamy, J. Wibowo, and A. Tordesillas, Phys. Rev. E 72, 041307 (2005).

[46] E. Aharonov and D. Sparks, Phys. Rev. E 60, 6890 (1999).

[47] A. Smart and J. M. Ottino, Soft Matter 4, 2125 (2008).

[48] D. M. Mueth, H. M. Jaeger, and S. R. Nagel, Phys. Rev. E 57, 3164 (1998).

[49] A. R. T. van Eerd, W. G. Ellenbroek, M. van Hecke, J. H. Snoeijer, and T. J. H. Vlugt, Phys. Rev. E 75, 060302 (2007).

[50] A. R. T. van Eerd, B. P. Tidhe, and T. J. H. Vlugt, Mol. Simul. 35, 1168 (2009).

[51] C. S. O'Hern, S. A. Langer, A. J. Liu, and S. R. Nagel, Phys. Rev. Lett. 86, 111 (2001).

[52] L. E. Silbert, D. Ertas, G. S. Grest, T. C. Halsey, and D. Levine, Phys. Rev. E 65, 051307 (2002).

[53] E. I. Corwin, H. M. Jaeger, and S. R. Nagel, Nature (London) 435, 1075 (2005).

[54] F. da Cruz, S. Emam, M. Prochnow, J.-N. Roux, and F. Chevoir, Phys. Rev. E 72, 021309 (2005).

[55] K. J. Dong, R. Y. Yang, R. P. Zou, X. Z. An, and A. B. Yu, Europhys. Lett. 86, 46003 (2009).

[56] A. J. Liu and S. R. Nagel, Nature (London) 396, 21 (1998).

[57] M. van Hecke, J. Phys.: Condens. Matter 22, 033101 (2010).

[58] C. S. O'Hern, S. A. Langer, A. J. Liu, and S. R. Nagel, Phys. Rev. Lett. 88, 075507 (2002).

[59] C. S. O'Hern, L. E. Silbert, A. J. Liu, and S. R. Nagel, Phys. Rev. E 68, 011306 (2003).

[60] T. S. Majmudar, M. Sperl, S. Luding, and R. P. Behringer, Phys. Rev. Lett. 98, 058001 (2007).

[61] A. J. Liu and S. R. Nagel, Annu. Rev. Condens. Matter Phys. 1, 347 (2010).

[62] L. E. Silbert, Soft Matter 6, 2918 (2010).

[63] M. P. Ciamarra and A. Coniglio, Phys. Rev. Lett. 103, 235701 (2009). 